\title{
Direct Reaction Theory for Exotic Nuclei
}

\author{
Angela Bonaccorso*t \\ Istituto Nazionale di Fisica Nucleare, Sezione di Pisa, 56127 Pisa, Italy. \\ E-mail: bonac@df.unipi.it
}

\begin{abstract}
This paper is organized around two main themes: exotic nuclei and direct reactions. The structure of exotic nuclei has been studied only from around 1985, because they are very short lived and before that it was not possible to produce and deliver them as beams on a target. They have large $\mathrm{N} / \mathrm{Z}$ or $\mathrm{Z} / \mathrm{N}$ ratios, are weakly bound and quite extended most of the times. Thus breakup, transfer and/or inelastic excitations of the surface are some of their most common reaction mechanisms. On the other hand direct reactions have been studied and understood for a much longer time, starting with the pioneering experiments in the early '50 on deuteron induced reactions and the reaction models developed by S.T. Butler and collaborators. Clearly direct reaction theory began thanks to the contribution of several Australian physicists and it is very appropriate to discuss it in the present context, together with its most recent developments in the field of exotic nuclei. Both subjects are extremely vast and I will discuss here only a few examples of the many interesting problems that have been encountered and solved in all those years. The choice is very personal and it is based solely on my research experience.
\end{abstract}

The 26th International Nuclear Physics Conference

11-16 September, 2016

Adelaide, Australia

\footnotetext{
* Speaker.

${ }^{\dagger}$ The original work presented here has been made in collaboration with G. Blanchon, D. M. Brink, F. Carstoiu, R. J. Charity, A. Garcia-Camacho, R. Kumar, G. Salvioni , N. Vin Mau and the MAGNEX group.
} 
For the last thirty years Nuclear Physics has known a big revival thanks to the advent of Radioactive Ion Beams (RIBs). These nuclei are often called "exotic" because they have unusual properties, like large neutron over proton ratios, N/Z; small separation energies for the valence particles; large root mean square radii, r.m.s. , and of course being radioactive they have first of all very short life times (for example ${ }^{11} \mathrm{Be}$ has a $\mathrm{T}_{1 / 2}=13.8 \mathrm{sec}$ while ${ }^{11} \mathrm{Li}$ has a $\mathrm{T}_{1 / 2}=8.6 \mathrm{~ms}$ ).

The most exotic nuclei are those just beyond the dripline. They are unbound but their ground state is often a resonance that lives long enough for its properties to be measured. The same might happen for the lowest excited states and thus these exotic systems are called "nuclei" and their properties studied in the same way as the more "normal" species that lie more or less closer to the stability line. Exotic nuclei are very weakly bound in most of the cases. Because of this their surface is quite extended. Thus several of their new properties have been discovered thanks to experiments employing peripheral, direct reactions. Such field is so rich and active that since 1999 there is a biennial conference called DREB (Direct reactions with Exotic Beams) in which a large number of physicists gather to discuss the latest developments. One special aspect of this conference is that the talks are mostly given by very young researchers, often in the stage of doing a post-doc or even a PhD thesis. The latest edition was held in Halifax, Canada http://conferences.triumf.ca/DREB2016/index.html.

The physics of exotic nuclei has shed a completely new light on the concept of the nuclear interaction and the way it acts to bind matter. The basic concepts of Nuclear Physics, such as nuclear radii, binding energies, decay schemes etc. have been rediscussed. So much so that it has been deemed necessary to organize schools for undergraduates in which these new concepts not contained in standard Nuclear Physics textbooks have been presented. One such a School, which has been held twice so far in Pisa, Italy, is indeed called "Re-writing Nuclear Physics Textbooks..." ( see https://agenda.infn.it/conferenceDisplay.py?confId=12522).

In the following we will review the main steps in the history of direct reactions. It seems that there is a twenty to thirty year recurrent time span between fundamental turning points in such a history. The first twenty five years or so were dominated by deuteron and light nuclei induced reactions (1950-1975) then heavy-ions started to be used as projectiles following the advent of new, more powerful accelerators, finally from 1985 up-to-date exotic nuclei have dominated the field of Nuclear Physics with hadronic probes.

\section{How it all began}

In the book of Norman K. Glendenning, " Direct Nuclear Reactions" [1] we can read about the beginning of nuclear reaction theory when it was thought that all reactions proceeded via compound nucleus [2] and resonance phenomena. They were first studied by Feshbach and Weisskopf [3], Breit and Wigner [4] and Kapur and Peierls [5]. Resonances are nowadays very important to study unbound nuclei and an updated review can be found in R. J. Charity contribution to the Pisa Summer School [6].

In the book of Glendenning there is also a nice figure from P. Hodgson book [7], that we report here as Fig. 1, in which every reaction seems to go through "compound nucleus" apart from direct reactions which are indicated to go to "...etc". In 1950 the first experiments [8] and theoretical papers [9] clarified what that "...ect" meant. From the point of view of the experimental observa- 
tions, direct reactions were characterized by forward peaked angular distributions and oscillations. Compound nucleus reactions had instead isotropic angular distributions. Bethe and Butler [10] first understood the mechanism of direct reactions as due mainly to a surface diffractive effect and showed the way to use them as spectroscopic tools. In fact the oscillations were different depending on the angular momenta involved (both initial and final state and relative motion were important ingredients). Later the first paper using semiclassical method appeared [11]. Direct reaction theory was born and its creche was in Australia! Some interesting aspects of deuteron induced reactions that were clarified are: the importance of matching the initial, final and relative motion energies as well as angular momenta and the fact that one particle acted as a spectator. The probability of such a mechanism was enhanced because the deuteron is weakly bound.

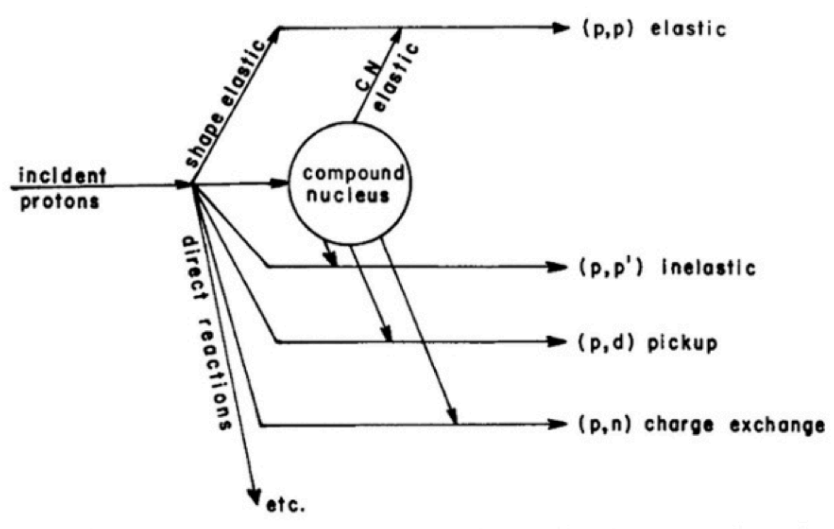

Figure 1: Illustration of proton-nucleus interaction mechanisms. [1]

Later on in the ' 70 with the advent of new, more powerful accelerators, ions heavier than deuterons and $\alpha$-particles were accelerated. Because those reactions were very forward peaked and the projectiles were now heavy, the angular distributions were often featureless. Then it became important to find new observables to measure, and the absolute values of transfer reactions were chosen to be fitted, for example, in order to get spectroscopic information such as the angular momentum of the initial single particle state and/or its spectroscopic factor. There was a paper that opened a long series of speculations [12]. It was shown that experimental absolute cross sections for transfer reactions such as ${ }^{12} \mathrm{C}+{ }^{12} \mathrm{C} \rightarrow{ }^{11} \mathrm{C}+{ }^{13} \mathrm{C}$ increased at low energy till a maximum and then decreased quite steeply if the incident energy increased further. Von Oertzen [13] and later Brink $[14,15]$ and collaborators showed that this behavior was due to the fact that the transfer probability was mainly given by an overlap integral of the momentum distributions of the initial and final single particle states. When the kinematical conditions were such that the nucleon was picked from the top of its initial distribution and put on the top of the final distribution, the transfer probability had a maximum. The overlap of the distributions was largest when the classical condition was met $\left|\varepsilon_{i}-\varepsilon_{f}\right|=\frac{1}{2} m v^{2}$, where $\varepsilon_{i}$ and $\varepsilon_{f}$ are the initial and final single particle binding energies while $\frac{1}{2} m v^{2}$ is the incident energy per particle at the distance of closest approach for the trajectory of 
relative motion between projectile and target. Increasing or decreasing the incident energy from the optimal value lead to a decrease of the absolute cross section. For some time there was also another puzzle related to some oxygen induced reactions whose absolute cross sections were not reproduced by the newly written finite rage DWBA code Ptolemy [16]. In this period a series of papers, both experimental, as well theoretical, tried to clarify the content of the Distorted Wave Born Approximation [17] that in the meantime had become the standard theoretical tool to analyze transfer and inelastic excitation data. Later Winther and collaborators [18] developed an analytical method to disentangle the structure of the DWBA equations and they were able to show that it was of fundamental importance to use an energy dependent optical potential to describe the initial and final channel distorted waves. Again similar methods have echoed recently in the physics with exotic nuclei. See for example [19].

\section{Experimental data vs. reaction and structure theories}

Direct reactions involve few nucleons and few degrees of freedom but to "model" them requires understanding the whole nucleus and all other possible reactions. One typical example is elastic scattering and the optical potential necessary to describe it, see M. Borge contribution to this conference. It requires also the understanding of experimental setups and the handling of data to extract meaningful observables. Reaction theory has to be simple and transparent in its interpretation to help disentangling the physical processes and allow experimentalists to describe their data. Finally reaction theorists must understand stucture models ( see for example S. Bacca and Shan-Gui Zhou talks) and they must be able to develop models that describe data reliably and in a simple way.
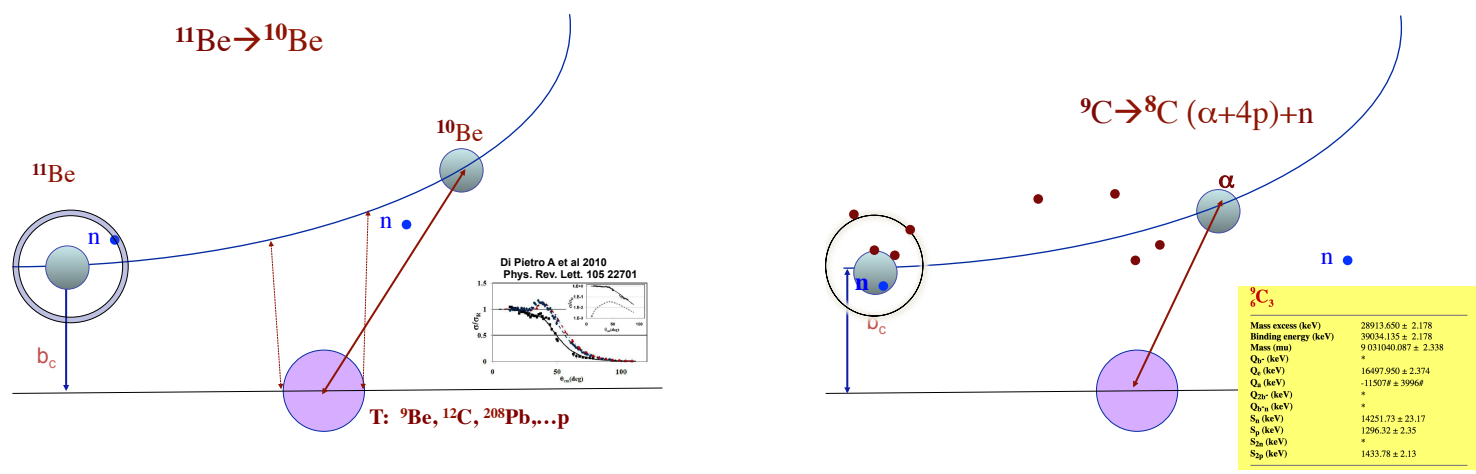

Figure 2: LHS: Scheme of a 1n-breakup reaction from ${ }^{11} \mathrm{Be}$ projectile in which the core ${ }^{10} \mathrm{Be}$ is left intact. RHS: 1n-brealup from ${ }^{9} \mathrm{C}$, in this case the "core", ${ }^{8} \mathrm{C}$ is an unbound nucleus which can be defined from the coincidence measurement of $\alpha+4 p$ particles in a charged particle detector such as the HiRA array [20].

To start the discussion on the direct reaction models we show in Fig. 2 two examples of one neutron breakup reactions from exotic nuclei. On the LHS a ${ }^{11}$ Be projectile, with a very small valence neutron separation energy $\mathrm{S}_{n}=0.5 \mathrm{MeV}$ is undergoing a scattering on a target which could be a heavy target like ${ }^{208} \mathrm{~Pb}$ or a light one like ${ }^{9} \mathrm{Be}$ or even a proton. We imagine the reaction is proceeding at hight enough energy to suppose a classical trajectory of relative motion. In the insert 


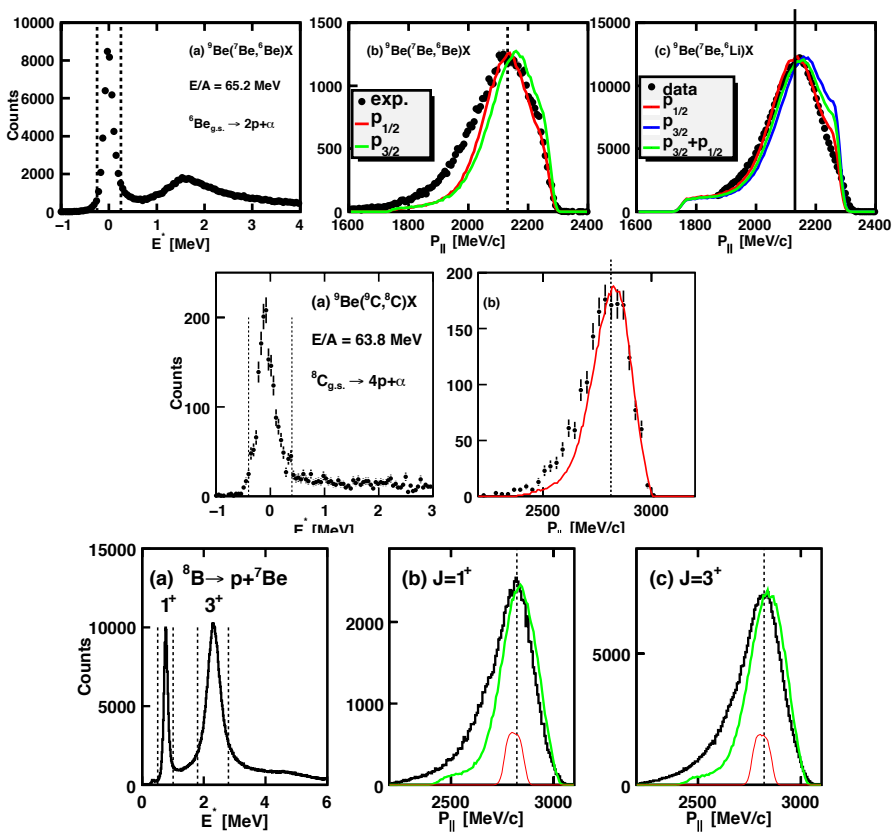

Figure 3: Top: Experimental ${ }^{6} \mathrm{Be}$ invariant-mass spectrum (a) and (b,c) the parallel-momentum distributions [21] of the reactions: ${ }^{9} \mathrm{Be}\left({ }^{7} \mathrm{Be},{ }^{6} \mathrm{Be}\right) \mathrm{X},{ }^{9} \mathrm{Be}\left({ }^{7} \mathrm{Be},{ }^{6} \mathrm{Li}\right) \mathrm{X}$ at mid-target energy of $65.2 \mathrm{AMeV}$. In all plots the dashed line on the $\mathrm{P}_{\|}$spectra indicates the momentum of the unreacted beam. The dashed lines in (a) show the gate on the ${ }^{6} \mathrm{Be}$ ground state. Middle: (a) The experimental ${ }^{8} \mathrm{C}$ invariant-mass spectrum and (b) the parallel-momentum distributions [21] of the reactions: ${ }^{9} \mathrm{Be}\left({ }^{9} \mathrm{C},{ }^{8} \mathrm{C}\right) \mathrm{X}$ at mid-target energy of $63.8 \mathrm{AMeV}$. The gate on the ground state of ${ }^{8} \mathrm{C}$ is indicated by the dashed lines in (a). Bottom: The experimental ${ }^{8} \mathrm{~B}$ invariant-mass spectrum for the $p+{ }^{7} \mathrm{Be}$ channels and $(\mathrm{b}, \mathrm{c})$ the parallel-momentum distributions [21] of the reaction ${ }^{9} \mathrm{Be}\left({ }^{9} \mathrm{C},{ }^{8} \mathrm{~B}_{1^{+}}\right) \mathrm{X}$ and ${ }^{9} \mathrm{Be}\left({ }^{9} \mathrm{C},{ }^{8} \mathrm{~B}_{3^{+}}\right) \mathrm{X}$ at $64.4 \mathrm{AMeV}$.

we show the elastic scattering angular distributions for ${ }^{11} \mathrm{Be}$ and ${ }^{10} \mathrm{Be}$ measured on a medium mass target [22]. One can see that ${ }^{11} \mathrm{Be}$ elastic scattering is depleted with respect to the case of a ${ }^{10} \mathrm{Be}$ projectile. If the target is heavy the weakly bound neutron would mainly be emitted because of a recoil of the ${ }^{10} \mathrm{Be}$ core from which an effective force originates which acts on the neutron. This is usually known as "Coulomb breakup" [23, 24]. Another interpretation is that the neutron feels the effect of a virtual photon [25]. If the target is light the neutron can be perturbed only by its nuclear interaction with the potential representing the target. On the RHS of Fig.2 a more complicated reaction is illustrated. Here a deeply bound neutron, $\mathrm{S}_{n}=14.25 \mathrm{MeV}$, is emitted from ${ }^{9} \mathrm{C}$. For this nucleus the valence proton separation energy is $\mathrm{S}_{p}=1.3 \mathrm{MeV}$, as shown in the insert from the nuclear data base [26]. The remaining "core" is ${ }^{8} \mathrm{C}$ which is un unbound nucleus. A coincidence measurement of an $\alpha$ plus four protons and the use of the invariant mass method [20] allow to reconstruct the spectrum of ${ }^{8} \mathrm{C}$, which is composed only of resonant states. By gating on each peak it is possible to reconstruct the momentum distribution of the center of mass of the $\alpha+4 p$ and then deduce the angular momentum of the initial state from its shape and width. Such a challenging type of experiment and the interpretation of the results are interesting in themselves and in Fig.3 we give a collection of examples from [21].

However there is also another reason to study ${ }^{9} \mathrm{C}$, as it is entering the nucleo-synthesis $p p$ - 
chain $[27,28]$. In fact one of the most interesting aspects of physics with Radioactive Ion Beams is the possibility to study on the earth some of the nuclei which are involved in the nucleo-synthesis of the elements and in particular in explosive nuclear burning where the cross sections of each nuclear reaction are extremely difficult to measure directly. For such cases, indirect and/or surrogate reactions can be used. One of the most intriguing case is the ${ }^{8} \mathrm{~B}(\mathrm{p}, \gamma)^{9} \mathrm{C}$ reaction which ignites the explosive hydrogen burning called hot $p p$ chain [27] or the ${ }^{7} \mathrm{Be}(\mathrm{p}, \gamma)^{8} \mathrm{~B}$ reaction [28]. The experimental determination of the ${ }^{8} \mathrm{~B}(\mathrm{p}, \gamma)^{9} \mathrm{C}$ cross section is also important for an accurate estimate of ${ }^{7} \mathrm{Li}$ synthesis. In fact in explosive hydrogen burning in novae, ${ }^{7} \mathrm{Li}$ can be synthesized through the $\beta$-decay of ${ }^{7} \mathrm{~B}$. On the other hand, the ${ }^{7} \mathrm{~B}(\mathrm{p}, \gamma)^{8} \mathrm{~B}$ reaction converts ${ }^{7} \mathrm{~B}$ to ${ }^{8} \mathrm{~B}$, while the inverse ${ }^{8} \mathrm{~B}(\gamma, \mathrm{p})^{7} \mathrm{~B}$ reaction reduces the amount of ${ }^{8} \mathrm{~B}$. Since the two reactions are in equilibrium, a decrease of ${ }^{8} \mathrm{~B}$ due to the ${ }^{8} \mathrm{~B}(\mathrm{p}, \gamma){ }^{9} \mathrm{C}$ reaction would lead to a reduction of ${ }^{7} \mathrm{~B}$, and hence to a lower production of ${ }^{7} \mathrm{Li}$ compared with the case where the contribution of the ${ }^{8} \mathrm{~B}(\mathrm{p}, \gamma){ }^{9} \mathrm{C}$ reaction is ignored.

What makes ${ }^{9} \mathrm{C}$ particularly intriguing from the reaction mechanism point of view is the fact that the one neutron separation energy is about a factor ten larger than the proton separation energy while the one and two proton separation energies are almost identical, which is one of the reasons for ${ }^{8} \mathrm{C}$ being unbound. The reactions involving the nuclei just described are perfect examples of most discussed reaction mechanisms vs. structure topics of present day physics with RIBs, namely: unbound nuclei, n-knockout from deeply bound states which involves reduced cross sections, elastic scattering, total reaction cross sections and the optical potentials necessary to calculate them. We proceed then to discuss such points.

\section{A consistent treatment of transfer and breakup reaction mechanisms}

Nucleon transfer between bound states and nucleon removal can be described by the semiclassical model of Brink and collaborators $[14,15]$. The model was introduced to describe transfer between bound states and later on was generalized as transfer-to-the-continuum (TC) model to describe final continuum states. It was introduced for stable, well-bound nuclei [29] and revised recently $[30,31]$ on several occasions. The model is mostly analytical and it was originally thought to be able only to describe gross features of high energy reactions. The main aim was to disentangle the various physical ingredients entering a standard DWBA calculation. Because of its original simplicity several improvements have been possible. In its latest version, it has been modified to take into account the competing breakup channels present when one measures neutron knockout from nuclei where the valence neutron separation energy is much larger than the proton value. A term $e^{-P_{-p}} \approx 1-P_{-p}\left(b_{c}\right)$, has been introduced which is the probability that the weakly-bound proton in the projectile does not itself get knocked out in the collision. An equivalent term can also be used when knocking out a proton from a neutron-rich system where the valence neutrons are weakly bound. This is equivalent to calculating a Dynamic Polarization Potential (DPP) from the phase shift [32]. Thus most of the effects found in knockout of nuclei with large differences in valence neutron and proton separation energies should not be unexpected in view of the fact that one is measuring a weakly-populated channel which is clearly in competition with another which has a much higher population probability. 
The differential cross section for a process in which a strongly-bound neutron is knocked out, but a weakly-bound proton is not, is given as

$$
\frac{d \sigma_{T C}^{n o p}}{d \xi}=(A N C)^{2} \int_{0}^{\infty} d \mathbf{b}_{\mathbf{c}}\left|S_{c t}\left(b_{c}\right)\right|^{2} e^{-P_{-p}} \frac{P_{-n}\left(b_{c}\right)}{d \xi},
$$

where $\xi$ can be equal to $\varepsilon_{f}$, the final continuum energy of the nucleon with respect to the target, or to $k_{1}$, the z-component of the nucleon momentum in the initial state, or to the final core parallel momentum $P_{\|}$defined below. The total breakup cross section is obtained by integrating the differential cross section over $\xi$. There is also an integral on the core-target impact parameter weighed by the probability $\left|S_{c t}\left(b_{c}\right)\right|^{2}$ that the core has survived the scattering "intact" and also that the weakly-bound particle has not been knocked out and absorbed by the target. (ANC) is the asymptotic normalization constant as given in [33] in the case of (VMC) initial wave functions which are normalized to the spectroscopic factor. In the case of Woods-Saxon wave functions normalized to unity, it is the product of the square root of a shell-model spectroscopic factor and

$$
C_{i}=\lim _{r \rightarrow \infty} \psi_{\text {num }}(r) /\left(i \gamma h_{l}^{(1)}(i \gamma r)\right)
$$

the initial wave-function asymptotic normalization constant given as the ratio of the numerical wave function and the Hankel function [29], where $\gamma=\sqrt{2 m S_{n}} / \hbar$ is the decay length of the initial wave function and $S_{n}$ is the nucleon separation energy.

The breakup probability in the projectile reference frame is

$$
\frac{d P}{d k_{1}} \approx \frac{1}{2} \Sigma_{j_{f}}\left(\left|1-\bar{S}_{j_{f}}\right|^{2}+1-\left|\bar{S}_{j_{f}}\right|^{2}\right)\left(2 j_{f}+1\right)\left(1+R_{i f}\right)\left[\frac{\hbar}{m v}\right] \frac{1}{k_{f}}\left|C_{i}\right|^{2} \frac{e^{-2 \eta b_{c}}}{2 \eta b_{c}} M_{l_{f} l_{i}} .
$$

where $\bar{S}_{j_{f}}$ are neutron-target S-matrices calculated according to the optical model, including the spin-orbit term of the neutron-target optical potential. The sum over partial waves in Eq.(3.2) is indeed a sum over total neutron-target angular momenta. $C_{i}=1$ if the (ANC) from [33] is used in Eq.(3.1). The form factor $\frac{e^{-2 \eta b_{c}}}{2 \eta b_{c}}$ is due to the combined effects of the Fourier transforms of the initial and final wave-functions, while $M_{l_{f} l_{i}}$ is due the to overlap of their angular parts and $\mathrm{R}_{i f}$ are spin-coupling coefficients. More detailed definitions and discussions are given in Ref.[30, 34].

\subsection{Kinematics of knockout}

In knockout experiments, it is assumed that a measure of the residual-nucleus (core) $P_{\|}$spectrum gives a direct information on the momentum distribution of the valence particle in the initial state of the projectile. This is possible only if one assumes that the transfer of energy into perpendicular momentum is negligible.

Define $T_{p}$ and $T_{r}$ as the projectile and residual kinetic energies and $M_{p}, M_{r}$, and $M_{t}$ as the projectile, residual (core), and target masses, respectively. The other kinematical variables involved in the process are $k_{1}=\left(\varepsilon_{f}-\varepsilon_{i}-\frac{1}{2} m v^{2}\right) /(\hbar v)$, the z-components of the neutron momentum in the initial state, $-\varepsilon_{i}=S_{n} \equiv\left(M_{r}+M_{n}\right)-M_{p}$, the initial separation energy of the valence particle in the projectile, $\varepsilon_{f}$, the final energy with respect to the target, and $\frac{1}{2} m v^{2}$ is the incident energy per particle at the distance of closest approach. 
Eq.(3.2) is obtained from a first-order time-dependent amplitude which assumes that the transfer of energy from the projectile to the target is only due to the knocked out particle. Furthermore it is assumed that the perpendicular component of the neutron momentum $k_{\perp}=i \eta$ is conserved. Notice, $\eta$ is the parameter which gives the slope of the form factor in Eq.(3.2) and eventually the shape of the spectrum. Thus, the transfer of energy is all converted into the change of nucleon parallel momentum according to the above definitions. The core parallel momentum is given by

$$
P_{\|}=\sqrt{\left(T_{r}+M_{r}\right)^{2}-M_{r}^{2}}
$$

By applying energy conservation between initial and final states $M_{p}+T_{p}+M_{t}=T_{r}+M_{r}+M_{n}+$ $M_{t}+\varepsilon_{f}$, then if the target mass is conserved $\left(\varepsilon_{f}<M_{t}\right), M_{t}$ cancels out and $T_{r}=T_{p}+M_{p}-$ $\left(M_{r}+M_{n}\right)-\varepsilon_{f}=T_{p}+\varepsilon_{i}-\varepsilon_{f}$ where $\varepsilon_{i}$ the valence-neutron binding energy in the projectile defined above. Under the eikonal hypothesis that energy is converted mainly in parallel momentum, then by inserting the previous expression for $T_{r}$ in Eq.(3.3), it follows that the ejectile parallel momentum is given by:

$$
P_{\|}=\sqrt{\left(T_{p}+\varepsilon_{i}-\varepsilon_{f}\right)^{2}+2 M_{r}\left(T_{p}+\varepsilon_{i}-\varepsilon_{f}\right)} .
$$

Using this and the relative Jacobian, the core parallel-momentum distribution in the laboratory can be obtained from Eq.(3.2) which is directly comparable to the measured momentum distributions. Notice that the maximum final nucleon target energies involved are typically around $2 \mathrm{E}_{\text {inc }}$ with $\mathrm{E}_{\text {inc }} \approx 65 \mathrm{AMeV}$ for the experiments discussed here. In some cases, in order to reproduce the long tails of the spectra, we have calculated up to $\varepsilon_{f}=240 \mathrm{MeV}$. Thus the condition $\varepsilon_{f}<M_{t}$ is well satisfied for a ${ }^{9} \mathrm{Be}\left(\mathrm{M}_{t}=8385.2 \mathrm{MeV}\right)$ target and would be satisfied even for a target as small as the deuteron, while it is less satisfied at relativistic incident energies. With this we have demonstrated that the statement contained in [35] about the inaccuracy of our prescription Eq.(3.4), is unjustified.

The original formulation of the TC method is for neutrons as its development goes through several analytical steps that can be performed only using the asymptotic (Hankel) form of the initial wave function. However in Ref. [36] we have shown that a neutron wave function corresponding to an effective separation energy $\left(S_{n}^{\text {eff }}\right)$ can always be fitted to the exact proton wave function and that the nuclear-breakup observables thus calculated within the eikonal model are indistinguishable.

\subsection{Constraining the (nucleus-nucleus) S-matrix by total reaction cross section calculations vs experimental data}

The potentials used to calculate the energy-dependent S-matrix appearing in Eq.(3.1) are the $n-{ }^{9} \mathrm{Be}$ optical-model potentials recently developed by two methods in Ref. [37]. In one case (AB), the phenomenological potential consists of a Wood-Saxon real volume and a spin-orbit term, a correction $\delta \mathrm{V}$ necessary to take into account the surface-deformation effects, and an imaginary term with both surface and volume components. The other method used in Ref. [37] is the Dispersive Optical Model (DOM) [38]-[43] where there are the same terms as in the (AB) potential but for the correction $\delta \mathrm{V}$ to the real potential which arises naturally from the imaginary potential via a dispersion relation. Both potentials reproduce the experimental $n-{ }^{9} \mathrm{Be}$ elastic, reaction and total cross section at all energies, in particular the $p_{3 / 2}$ resonance at $\mathrm{E}_{l a b}=0.7 \mathrm{MeV}$ and the $d_{5 / 2}$ resonance at $\mathrm{E}_{l a b}=3.1 \mathrm{MeV}$. We show in Fig.4 the total reaction cross sections and the angular distributions 
from Ref. [37]. As one can see in both cases the agreement with the experimental data is striking, in particular if one considers that we are dealing with the optical potential for scattering on a light, very deformed nucleus.
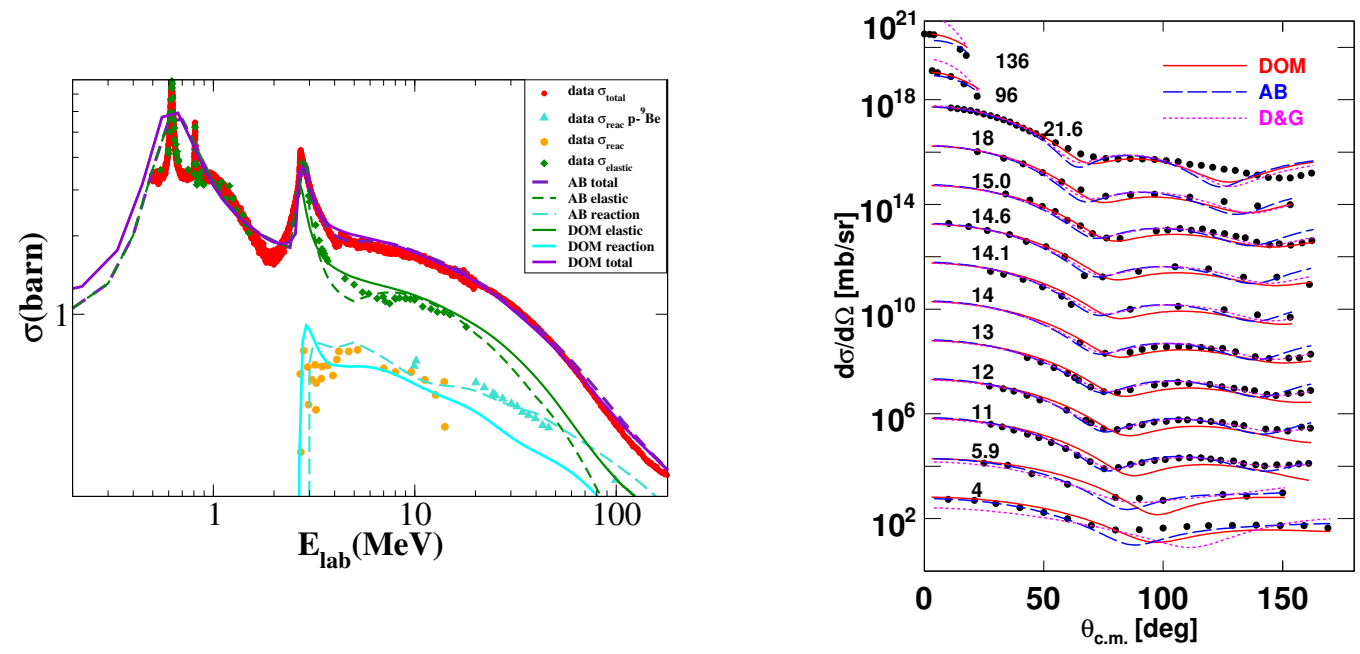

Figure 4: LHS: $n+{ }^{9} \mathrm{Be}$ cross sections calculated according to Ref.[37] and compared to the experimental data. RHS: Elastic scattering angular distributions.

By using Eq.(3.1), the core-target interaction is treated in the strong-absorption model which requires only the knowledge of the imaginary part of the core-target interaction. For consistency with our $n-{ }^{9} \mathrm{Be}$ optical potential, we have constructed [44] a single folded nucleus-nucleus potential using various core densities $\rho_{c}$ and the imaginary part $W^{n t}$ of the $(\mathrm{AB})$ potential discussed above.

This is possible, because in the Glauber description of nucleus-nucleus (core-target in our case) scattering, the reaction cross section is given by

$$
\sigma_{c t}^{R}=2 \pi \int_{0}^{\infty} b_{c} d b_{c}\left(1-\left|S_{c t}\left(\mathbf{b}_{\mathbf{c}}\right)\right|^{2}\right)
$$

where

$$
\left|S_{c t}\left(\mathbf{b}_{\mathbf{c}}\right)\right|^{2}=e^{-2 \chi_{I}\left(b_{c}\right)}
$$

can be interpreted as the probability that the scattering is elastic at a given impact parameter. The imaginary part of the eikonal phase shift can be written as

$$
\chi_{I}\left(\mathbf{b}_{\mathbf{c}}\right)=\frac{1}{\hbar v} \int d z W^{c t}\left(\mathbf{b}_{\mathbf{c}}, z\right)=\frac{1}{\hbar v} \int d^{2} \mathbf{b}_{\mathbf{1}} \int d z W^{n t}\left(\mathbf{b}_{\mathbf{1}}-\mathbf{b}_{\mathbf{c}}, z\right) \int d z_{1} \rho_{c}\left(\mathbf{b}_{\mathbf{1}}, z_{1}\right),
$$

where

$$
W^{c t}\left(\mathbf{r}_{\mathbf{c}}\right)=\int d^{3} \mathbf{r}_{\mathbf{1}} W^{n t}\left(\mathbf{r}_{\mathbf{1}}-\mathbf{r}_{\mathbf{c}}\right) \rho_{c}\left(\mathbf{r}_{\mathbf{1}}\right)
$$

is the imaginary part of the single folded optical potential given in terms of the imaginary part of the nucleon-nucleus optical potential $W^{n t}\left(\mathbf{r}_{\mathbf{c}}\right)$ and the matter density $\rho_{c}\left(\mathbf{b}_{\mathbf{1}}, z_{1}\right)$ of the core. $v$ is the nucleus-nucleus velocity of relative motion. According to Ref.[45], this procedure is justified for the imaginary part of the optical potential as the $(\mathrm{AB})$ and $(\mathrm{DOM})$ potentials are surface dominated. 
Suppose we had used instead a double folding model, as it is done by most of the existing knockout eikonal models. The phase shift would have been

$$
\chi_{I}(\mathbf{b})=-\frac{1}{2} \sigma_{n n} \int d \mathbf{b}_{\mathbf{1}} \int d z \rho_{t}\left(\mathbf{b}_{\mathbf{1}}-\mathbf{b}, z\right) \int d z_{1} \rho_{c}\left(\mathbf{b}_{\mathbf{1}}, z_{1}\right) .
$$

and the n-target potential

$$
W^{n t}(\mathbf{r})=-\frac{1}{2} \hbar v \sigma_{n n} \rho_{t}(\mathbf{r})
$$

would have been a single-folded zero-range $n$-target imaginary potential. In this case the $\mathrm{W}^{n t}$ potential would have the same range as the target density because $\sigma_{n n}$, the nucleon-nucleon cross section, is a simple scaling factor. With such a potential the distinction of surface and volume terms cannot be usually reproduced. In particular their respective contributions, which are strongly energy dependent, as shown by phenomenological potentials, cannot be distinguished. Indeed, Satchler and Love [45] discussing the folding model for ${ }^{9} \mathrm{Be}$ scattering, found evidence of anomalously large deformation and surface effects, which is consistent with the results of [37]. When experimental data are available, this problem is often solved by renormalizing the folded potential so that it would reproduce the data. However dynamical aspects of surface reactions which are typical and very relevant for light nuclei are difficult to reproduce even with a renormalized folded potential.

Our single folded potential presents thus several advantages with respect to double folding models:

- The imaginary potential is correctly second order because of the phenomenological nature of the $n$-target potential.

- The projectile density can be better tested because one is free from the ambiguity on the target density.

- The ambiguity on the nucleon-nucleon interaction to be used is overcome.

- The energy dependence of the potential is correctly reproduced because of the underlying correctness of the n-target potential.

- Deformation, surface modes of excitation and/or breakup of the target are correctly taken into account and one is left with the task of modeling the same effects for the exotic projectile.

The last point is taken into account by adding to the single-folding potential, a surface potential of the Woods-Saxon-derivative type.

$$
W(r)=-4 a^{i} W_{\text {surf }} \frac{d}{d r} \frac{1}{1+e^{\left(r-R^{i}\right) / a^{i}}} .
$$

with very small strength $\left(\mathrm{W}_{\text {surf }}=0.8\right.$ to $0.015 \mathrm{MeV}$ ). The radius has been taken [44] as $\mathrm{R}^{i}=0.92$ $\left(\mathrm{A}_{p}^{1 / 3}+\mathrm{A}_{t}^{1 / 3}\right)=3.8 \mathrm{fm}$, which is close to the distance of closest approach for head on collisions where the absorption is maximized. On the other hand the diffuseness should be large according to [21, 32], and equal to $a^{i}=1 /\left(2 \sqrt{2 m S_{p}} / \hbar\right)=2$ fm for ${ }^{9} \mathrm{C}$, since $\mathrm{S}_{p}=1.296 \mathrm{MeV}$. 


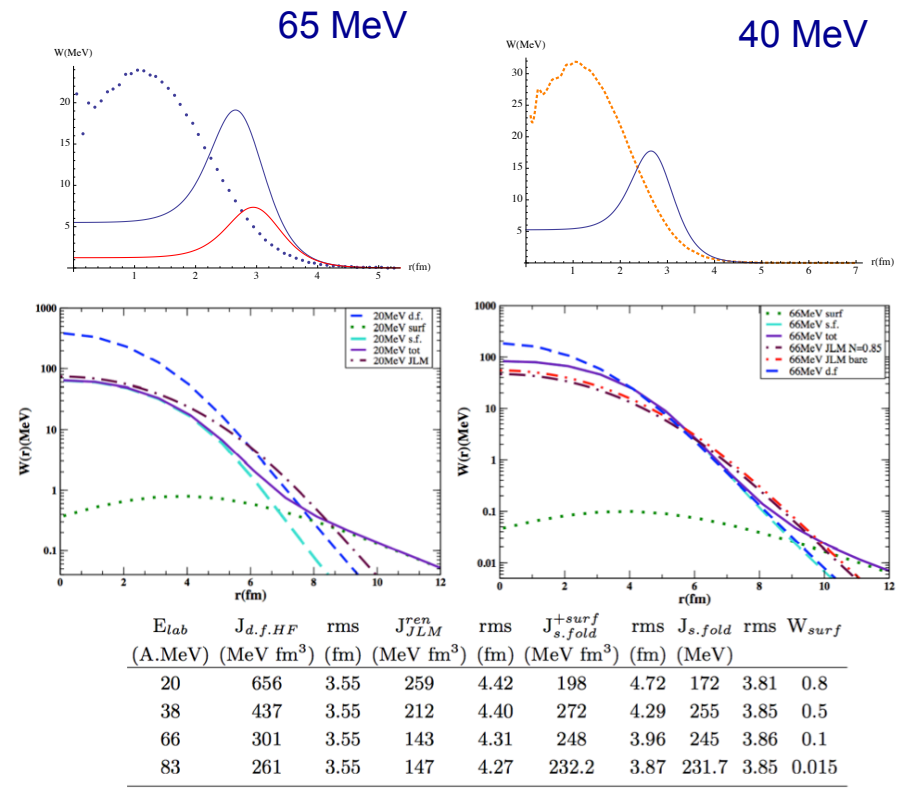

Figure 5: LHS: $n-{ }^{9} \mathrm{Be}$ single folded (dotted line) potential from Eq.(3.10) and phenomenological potentials at $65 \mathrm{MeV}$ from Ref.[37]. Red line is the DOM potential, blue line is the AB potential. RHS: The same at $40 \mathrm{MeV}$. Middle and bottom parts: imaginary potentials ${ }^{9} \mathrm{C}-{ }^{9} \mathrm{Be}$ and their volume integrals and r.m.s. from Ref.[44]. See text for details.

In Ref.[44] we have given a series of examples of potentials calculated with the single or double-folded potential and with the JLM method [46]. We report here in Fig.5, top part, the comparison between a $n-{ }^{9} \mathrm{Be}$ potential calculated with the parametrization of [37] or with the singlefolded model Eq.(3.10) using the ${ }^{9} \mathrm{Be}$ density from [47, 48]. Clearly the single-folded potential simply reproduces the shape of the density of the ${ }^{9} \mathrm{Be}$ target from Ref. [47] while the phenomenological potential has a shape which represents better the concentration of reaction channels on the surface. In the middle part of the figure we show the single folded ${ }^{9} \mathrm{C}-{ }^{9} \mathrm{Be}$ potentials obtained folding the $\mathrm{AB}$ potential of [37] with the HF density of ${ }^{9} \mathrm{C}$ from [44]. Finally the surface term given in equation (3.11) is added to provide the long tail which is necessary to take into account the surface reaction channels of a projectile such as ${ }^{9} \mathrm{C}$. In the bottom part of the figure we give for the same potentials the volume integrals and corresponding r.m.s. values.

We conclude this section showing in Fig.6 total reaction cross sections calculated in [44] for ${ }^{9} \mathrm{C}-{ }^{9} \mathrm{Be}$ scattering and data points from $[49,50,51]$. Our model reproduces very well the absolute values of the cross sections and their energy behavior. This is very important because it provides a method by which the density of the exotic projectile can be tested free from uncertainties from the target density. Thus the $\left|S_{c t}\right|^{2}$ calculated with our model and our potentials can be considered reliable and we have made a step forward in the accuracy of the calculations of breakup cross sections. It is evident in fact that if the double folding model gives too small reaction cross section, see the curve in Fig.6, the same method applied in the calculation of the core-target survival probabili- 

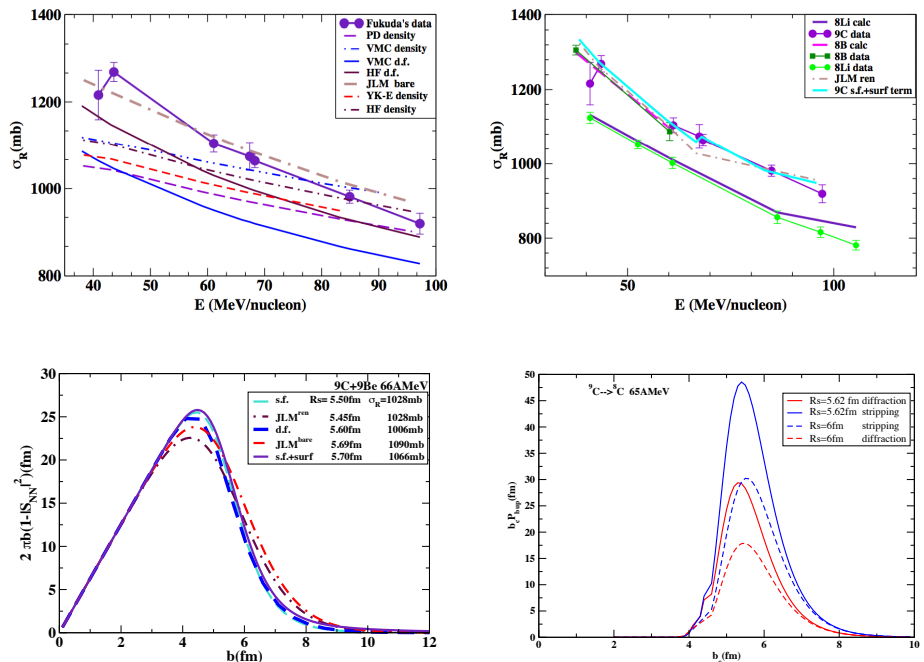

Figure 6: LHS, top: Energy dependence of the reaction cross sections for ${ }^{9} \mathrm{C}-{ }^{9} \mathrm{Be}$ calculated according to Eq.(3.5). We compare results obtained using the the single folded potential with $\mathrm{n}-{ }^{9} \mathrm{Be}$ from [37] and various projectile densities (see [37] ) and JLM [46]. The two full lines are the results of calculations with the double folded potentials with Hartree-Fock densities. Thick marron line is with HF densities, blu thin line with VMC densities [47]. Data are from Ref.[49]. RHS: We show here results from the single folded potential plus the additional surface term Eq.(3.11) and a renormalized JLM and again the data from Ref.[49]. For comparison results obtained for ${ }^{8} \mathrm{Li}$ and ${ }^{8} \mathrm{~B}$ projectiles from Ref.[21] using the single-folded plus surface term potential. Data are from Ref. [50, 51]. LHS, bottom: Integrand of the total reaction cross section equation. RHS, bottom: Integrand of the breakup reaction cross section equation.

ties, according to Eqs.(3.9,3.10) would provide too large breakup cross sections because somewhat small impact parameters would correspond to cores surviving with a non negligible probability. In the bottom part of Fig. 6 we show on the LHS the integrant of the reaction cross section formula Eq.(3.5) while on the RHS we show the integrand of the breakup probability formula Eq.(3.1). One sees clearly that breakup is strongly localized on the overlap surface of the two nuclei.

Therefore we can interpret the possible reduction in spectroscopic factors extracted from knockout of a deeply bound particle [35] as partially due to an inaccuracy in the $S_{c t}$ values when calculated via double-folded potentials. Single folded potentials might be a better choice if the $\mathrm{n}$-target potential is known sufficiently well as it is the case for ${ }^{9} \mathrm{Be}$. Another reason might be the neglect of the kinematical effects and energy-momentum conservation discussed in Sec. 3.1.

The second important point is that since the beginning of studies of the structure of exotic nuclei, total reaction cross section measurement have been a simple and reliable tool to deduce sizes of exotic nuclei and a probe for their density distributions. See the the pioneering papers of Tanihata [52]-[56] and collaborators and the reviews by A. Ozawa [57] and M. Fukuda and collaborators $[49,50,51]$. Our single-folded potential method constitutes a step forward in understanding the shape of the matter distributions of exotic projectiles and its relationship with the way it shows up in a reaction. 


\section{Continuum states and unbound nuclei}
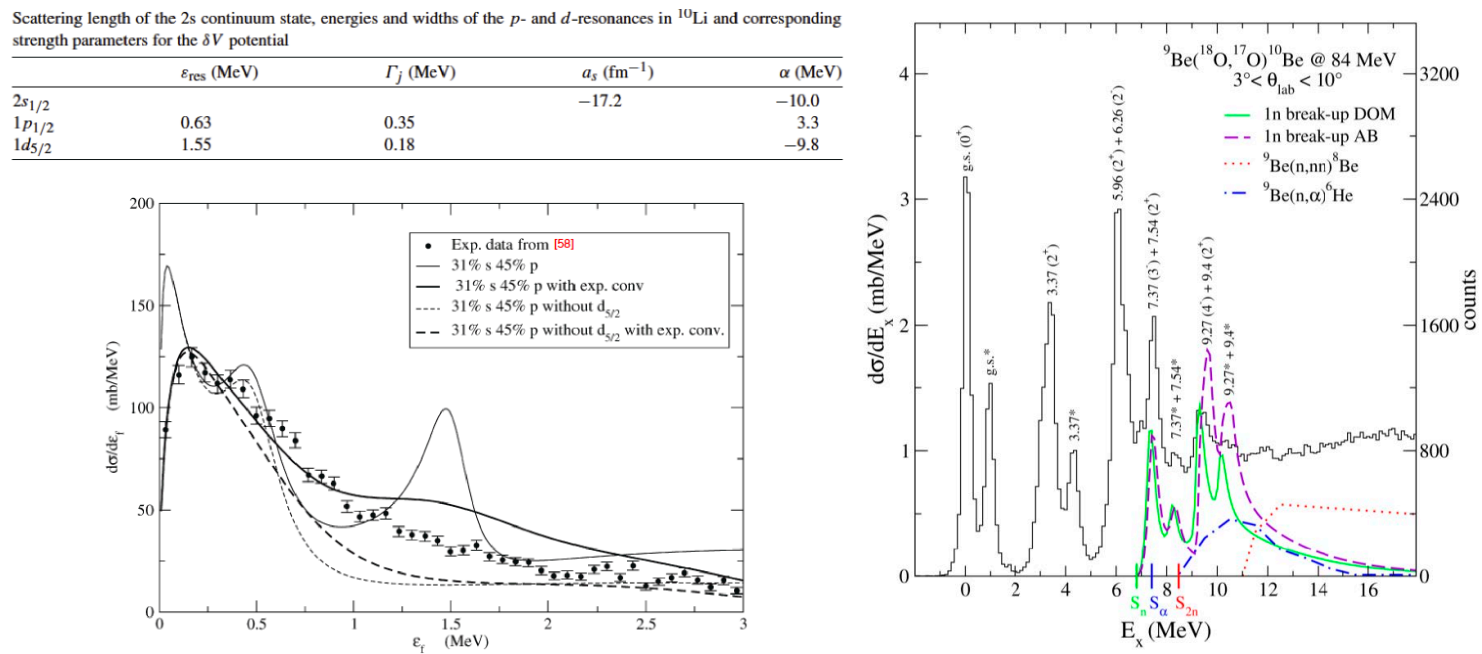

Figure 7: LHS: Relative energy spectrum $n-{ }^{9} \mathrm{Li}$ following ${ }^{11} \mathrm{Li}$ projectile fragmentation [61]. The table shows the structure inputs used for the s,p, and $\mathrm{d}$ final states. RHS: Inclusive excitation-energy spectrum of the ${ }^{9} \mathrm{Be}\left({ }^{18} \mathrm{O},{ }^{17} \mathrm{O}\right){ }^{10} \mathrm{Be}$ reaction at $84 \mathrm{MeV}$ incident energy [64]. Total $1 \mathrm{n}$ breakup calculations resulting from the use of the DOM and the $\mathrm{AB}$ potentials [37] are shown as the green continuous and the violet dashed lines, respectively. The renormalized experimental data of the ${ }^{9} \mathrm{Be}(\mathrm{n}, \mathrm{nn}){ }^{8} \mathrm{Be}[65]$ and ${ }^{9} \mathrm{Be}(\mathrm{n}, \alpha){ }^{6} \mathrm{He}[66]$ reactions are reported as red dotted and blue dotteddashed line, respectively. This is to show that they can contribute to the experimental background. The $1 \mathrm{n}-\left(\mathrm{S}_{n}\right), 2 \mathrm{n}-$ $\left(\mathrm{S}_{2 n}\right)$ and $\alpha-\left(\mathrm{S}_{\alpha}\right)$ separation energies are also indicated. Peaks marked with an asterisk refer to the ${ }^{17} \mathrm{O}$ ejectile emitted in its first excited state at $0.87 \mathrm{MeV}$.

An important aspect in the study of exotic nuclei is the understanding of spectra containing low lying resonances and the theoretical description of them. We have shown already in Fig. 3 a series of data and calculations from Ref. [21] which refer to proton unbound nuclei. They can be created by knockout of a deeply bound neutron from a projectile which is along the proton drip line. The invariant mass spectra are shown together with the parallel momentum spectra obtained by gating on the ground state resonance. For those nuclei we have not calculated the invariant mass spectra while the parallel momenta calculations were done with the formalism described in Sec. 3 and one can see that they agree very well with the data. The absolute cross sections are very sensitive to the way the $\left|S_{c t}\right|^{2}$ term is calculated and we have seen [21] that using the single folded potential Eq.(3.8) the knock out cross section for ${ }^{9} \mathrm{Be}\left({ }^{9} \mathrm{C},{ }^{8} \mathrm{C}\right) \mathrm{X}$ reduces by about a factor three while increasing the strong absorption radius by $6 \%$. Indeed the very small experimental cross section of $3.82 \mathrm{mb}$ could be obtained if the strong absorption radius was set to $\mathrm{R}_{s}=6.7 \mathrm{fm}$ corresponding to a radius parameter $\mathrm{r}_{s}=1.61 \mathrm{fm}$, where $\mathrm{R}_{s}$ is defined by $\left|S_{c t}\left(R_{s}\right)\right|^{2}=1 / 2$ and $\mathrm{R}_{s}=r_{s}\left(A_{p}^{1 / 3}+A_{t}^{1 / 3}\right)$. This is perhaps not unreasonable considering that ${ }^{8} \mathrm{C}$ is defined by the coincidence measurement of $(\alpha+4 p)$ in which all five particles must have survived an absorptive breakup on the ${ }^{9} \mathrm{Be}$ target.

The invariant mass spectra can be calculated with a method similar to to the TC, the only difference is that the final state is a continuum state with respect to the core of origin rather than the target [59]-[62]. We have successfully applied such a method to the study of ${ }^{10} \mathrm{Li}$ and ${ }^{13} \mathrm{Be}$ which are unbound sub-costituents of the borromean nuclei ${ }^{11} \mathrm{Li}$ and ${ }^{14} \mathrm{Be}$. In Fig.7 LHS, experimental 
data and our calculations are shown for ${ }^{10} \mathrm{Li}$. They are in very good agreement, furthermore a more recent experiment [63] has confirmed our values for the energy and width of the p-state resonance.

Another very intriguing nucleus is ${ }^{10} \mathrm{Be}$, the core of ${ }^{11} \mathrm{Be}$. It is itself a radioactive nucleus and neutron scattering data on it do not exist. It has been studied mainly by $\beta$-decay and neutron transfer. A recent experiment ${ }^{9} \mathrm{Be}\left({ }^{18} \mathrm{O},{ }^{17} \mathrm{O}\right){ }^{10} \mathrm{Be}$ [64] has provided the spectrum for both the bound and some unbound states. It is shown in Fig.7 RHS, together with the calculations made using the $\mathrm{n}-{ }^{9} \mathrm{Be}$ optical potential of Ref.[37] within the TC model of Sec.3. The agreement is very good and once again it shows the powerfulness of the TC to describe both projectile characteristics such as the parallel momentum distributions of Fig. 3 as well as target resonances as in Fig. 7.

\section{Conclusions and outlook: What next for 2017-2050?}

We have seen that the use of direct reaction experiments has been of great importance for the understanding of nuclear structure since the ' 50 and in particular in the last thirty years, with the advent of beams of radioactive nuclei. From the examples given in this paper, we can then draw the following conclusions and make some predictions for future developments:

- Nuclei can have larger sizes that predicted by formulae of standard textbooks with a radius parameter depending on the beam incident energy of the reaction used to fix it. We can have $\mathrm{r}_{s}=1.4 \mathrm{fm}$ at around $20 \mathrm{AMeV}$. Also imaginary parts of optical potential can have very large diffuseness, $\mathrm{a}=\frac{1}{2 \gamma}=3.2 \mathrm{fm}$ for a ${ }^{11} \mathrm{Be}$ induced reaction, obtained as twice the inverse of the valence nucleon wave-function decay length $[22,32]$.

- Surface effects, clustering and unbound structures are now under control.

- Structure and reaction models are being unified via ab-initio methods and full inclusion of continuum spectra (cf S. Bacca talk, and see also Dispersive Optical Model [38]-[43]).

- We still need to understand at a microscopic level the energy dependence of nn and NN interactions, including optical potentials.

- In the future will we study mainly unbound nuclei via resonance definition, similarly to what is done in elementary particle physics? (cf. W. Nazarewicz talk).

- Will improvements in numerical techniques allow to solve the nuclear many body problem "exactly"? and/or will semiclassical-analytical methods survive?

In this paper I have only discussed simple analytical formalisms to describe direct reactions. There are many groups using instead numerical approaches such as improved DWBA, Continuum Discretized Coupled Channel (CDCC), R-matrix formalism etc. Details on such methods can be found in Refs.[67, 68].

\section{References}

[1] Norman K. Glendenning, Direct Nuclear Reactions (Academic Press, New York,1983).

[2] N. Bohr, Nature 137344 (1936). 
[3] H. Feshbach and V. F. Weisskopf, Phys. Rev. C 761559 (1949). H. Feshbach, D. C. Peasle and V. F. Weisskopf, Phys. Rev. C 71145 (1947). H. Feshbach, C. E. Porter and V. F. Weisskopf, Phys. Rev. C 96448 (1954).

[4] G. Breit and E. P.Wigner, Phys. Rev. C 49519 (1936).

[5] P. L. Kapur and R. Peierls, Proc. R. Soc. (London) A 166277 (1938).

[6] R. J. Charity, Eur. Phys. J. Plus 13163 (2016).

[7] P. Hodgson, Nuclear Reactions and Nuclear Structure, (Clarendon Press, Oxford 1971).

[8] H. B. Borrows, W. M. Gibson and J. Rotblat, Phys. Rev. 801095 (1950).

[9] S. T. Butler, Phys. Rev. 801095 (1950).

[10] H. Bethe and S. T. Butler, Phys. Rev. 851045 (1952).

[11] S. T. Butler, N. Austern and C. Pearson, Phys. Rev. 1121227 (1958).

[12] J. S. Winfield et al., Phys. Lett. B 203 (1988).

[13] W. Von Oertzen, Phys. Lett. B 15195 (1985).

[14] L. Lo Monaco and D. M. Brink, J. Phys. G 11935 (1985).

[15] A. Bonaccorso, D. M. Brink and L. Lo Monaco, J. Phys. G 131407 (1987).

[16] S.C. Pieper et al., Phys. Rev. C 18180 (1978); C. Olmer et al., Phys. Rev. C 18205 (1978).

[17] K. Watson, Phys. Rev. 881163 (1952); M. Gell-Mann and M. L. Goldberger, Phys. Rev. 91398 (1953). J. Phys. G 131407 (1987).

[18] J. M. Quesada, A. Winther, Nucl. Phys. A 442381 (1985). J. H. Sørensen, A. Winther, G. Pollarolo, Nucl. Phys. A 522578 (1991).

[19] P. G. Hansen and J. A. Tostevin, Annu. Rev. Nucl. Part. Sci. 53219 (2003).

[20] R. J. Charity and the HiRA collaboration, Phys. Rev. C 84014320 (2011).

[21] A. Bonaccorso, R. J. Charity, R. Kumar and G. Salvioni, AIP Conf. Proc. 164530 (2015).

A. Bonaccorso, F. Carstoiu, R. J. Charity, R. Kumar, G. Salvioni, Few-Body Syst. 57331 (2016).

[22] A. Di Pietro et al., Phys. Rev. Lett. 10522701 (2010).

[23] J. Margueron, A. Bonaccorso and D. M. Brink. Nucl. Phys. A 703105 (2002).

[24] J. Margueron, A. Bonaccorso and D. M. Brink. Nucl. Phys. A 720337 (2003).

[25] C. A. Bertulani and G. Baur, Nucl. Phys. A 480615 (1988).

[26] http://nuclear.lu.se/database/masses/

[27] M. Wiescher, J. Görres, S. Graff, L. Buchmann, and F.-K. Thielemann, Astrophys. J. 343352 (1989).

[28] T. Motobayashi, Nucl. Phys. A 718 101c (2003), and private communication.

[29] A. Bonaccorso and D. M. Brink, Phys. Rev. C 38, 1776 (1988) and references therein.

A. Bonaccorso and D. M. Brink, Phys. Rev. C 43299 (1991).

[30] A. Bonaccorso, Physica Scripta T 152014019 (2013).

[31] A. Bonaccorso, JPS Conference Proceedings 6010023 (2015). 
[32] A. Bonaccorso and F. Carstoiu, Nucl. Phys. A 706322 (2002).

[33] K. M. Nollett and R. B. Wiringa, Phys. Rev. C 83 041001(R) ( 2011).

[34] A.Bonaccorso. Nucl. Phys. A 649 315c (1999).

A.Bonaccorso. Phys. Rev. C 60054604 (1999).

[35] J. A. Tostevin, A. Gade, Phys. Rev. C 90057602 (2014).

[36] Ravinder Kumar and Angela Bonaccorso. Phys. Rev. C 84014613 (2011).

[37] A. Bonaccorso and R. J. Charity, Phys. Rev. C 89024619 (2014).

[38] C. Mahaux and R. Sartor, Adv. Nucl. Phys. 201 (1991).

[39] R. J. Charity, L. G. Sobotka, and W. H. Dickhoff, Phys. Rev. Lett. 97162503 (2006).

[40] R. J. Charity, J.M.Mueller, L. G. Sobotka, andW. H. Dickhoff, Phys. Rev. C 76044314 (2007).

[41] J. M. Mueller, R. J. Charity, R. Shane, L. G. Sobotka, S. J. Waldecker, W. H. Dickhoff, A. S. Crowell, J. H. Esterline, B. Fallin, C. R. Howell, C. Westerfeldt, M. Youngs, B. J. Crowe, III, and R. S. Pedroni, Phys. Rev. C 83064605 (2011).

[42] W. H. Dickhoff and C. Barbieri, Prog. Part. Nucl. Phys. 52377 (2004).

[43] C. Barbieri, Phys. Rev. Lett. 103202502 (2009).

[44] A. Bonaccorso, F. Carstoiu, R. J. Charity, Phys. Rev. C 940346042016.

[45] G. R. Satchler and W. G. Love, Phys. Rep. 55183 (1979).

[46] J. P. Jeukenne, A. Lejeune, and C. Mahaux, Phys. Rev. C 1680 (1977).

[47] R. B. Wiringa, http://www.phy.anl.gov/theory/research/density/

[48] S. C. Pieper and R. B. Wiringa, Annu. Rev. Nucl. Part. Sci. 51, 53 (2001).

[49] M. Fukuda et al., private communication, also D. Nishimura et al., Osaka Univ. Lab. of Nuclear Studies (OULNS) Annual Report 2006, 37.

[50] G. W. Fan et al., Phys. Rev. C 90, 044321 (2014).

[51] M. Fukuda et al., Nucl. Phys. A 656209 (1999).

[52] I . Tanihata et al., Phys. Lett. B 160, 380 (1985).

[53] I. Tanihata et al., Phys. Rev. Lett. 55 (1985) 2676.

[54] I. Tanihata, Prog. Part. Nucl. Phys. 35505 (1995).

[55] T. Kobayashi et al. , Phys. Rev. Lett. 602599 (1988).

[56] I. Tanihata, Nucl. Phys. A 685 80c (2001).

[57] A. Ozawa et al., Nucl. Phys. A 691, 599 (2001).

A. Ozawa, AIP Conf. Proc. 865, 57 (2006); http://dx.doi.org/10.1063/1.2398828

[58] H. Simon et al., Nucl. Phys. A 791267 (2007).

[59] G. Blanchon, A. Bonaccorso and N. Vinh Mau, Nucl. Phys. A 739259 (2004).

[60] G. Blanchon, A. Bonaccorso, D.M. Brink, A. García-Camacho and N. Vinh Mau. Nucl. Phys. A 784 49 (2007). 
[61] G. Blanchon, A. Bonaccorso and D.M. Brink and N. Vinh Mau. Nucl. Phys. A 791303 (2007).

[62] G. Blanchon, N. Vinh Mau, A. Bonaccorso, M. Dupuis, N. Pillet, Phys. Rev. C 82034313 (2010).

[63] A. Sanetullaev et al, Phys. Lett. B 755481 (2016).

[64] D. Carbone et al., Phys. Rev. C 90064621 (2014).

[65] I. Murata et al., Conf. on Nucl. Data for Sci. Technology 2999 (2007); D. M. Drake et al., Nucl. Science and Engineering 63401 (1977); H. C. Catron et al., Phys. Rev. 123218 (1961); G. J. FIsher, Phys. Rev. 10899 (1957).

[66] R. Bass, T. W. Bonner and H. P. Haenni, Nucl. Phys. 23122 (1961); P. H. Stelson and F. C. Campbell, Phys. Rev. 1061252 (1957); M. E. Battat and F. L. Ribe, Phys. Rev. 8980 (1953).

[67] S. Typel, Eur. Phys. J. Plus 13113 (2016).

[68] A. Obertelli, Eur. Phys. J. Plus 131319 (2016). 